\title{
Conducting Drug Abuse Investigations in Natural Environments: Potential Directions for Medical Toxicology Research
}

\author{
Edward W. Boyer • Richard J. Fay • Aaron Cook • \\ Martin Buckosh • Patricia L. Hibberd • Patricia Case
}

Published online: 2 March 2010

(C) American College of Medical Toxicology 2010

\begin{abstract}
Physician-scientists, in the eyes of the National Institutes of Health (NIH), are crucial to the biomedical research enterprise since the development of evidence-based practice based on cutting-edge research. At the same time, NIH has heightened the importance of research mentorship by permitting investigators to revise an application a single time. The current NIH approach, therefore, narrows the margin of error allowable in a proposal and requires that investigators fully develop research protocols for initial submission. The purpose of this manuscript, therefore, is to provide medical toxicologists with a proven research methodology that can be applied to substance abuse investigations. A secondary aim is to provide successful grant language that can be used in subsequent applications for research funding.
\end{abstract}

Keywords Substance abuse - MSM - Adulterants · Coingestants $\cdot$ Club drugs

E. W. Boyer $(\bowtie) \cdot$ R. J. Fay $\cdot$ M. Buckosh

Division of Medical Toxicology, Department of Emergency

Medicine, University of Massachusetts Medical School,

55 Lake Avenue North,

Worcester, MA 01655, USA

e-mail: Edward.boyer@childrens.harvard.edu

\section{A. Cook}

Yale School of Public Health,

New Haven, CT, USA

P. L. Hibberd $\cdot$ P. Case

Tufts School of Medicine, Fenway Community Health,

Boston, MA, USA

\section{Introduction}

Physician-scientists, in the eyes of the National Institutes of Health $(\mathrm{NIH})$, are crucial to the biomedical research enterprise since the development of evidence-based practice based on cutting-edge research. At the same time, NIH has heightened the importance of research mentorship by permitting investigators to revise an application a single time. The current NIH approach, therefore, narrows the margin of error allowable in a proposal and requires that investigators fully develop research protocols for initial submission.

Most researchers conduct investigations related to their areas of expertise; for medical toxicologists, substance abuse investigations are an accessible academic area that is emphasized by NIH. The priorities of the $\mathrm{NIH}$, and the shortcomings of data obtained by poison control centers, suggest that medical toxicologists should expand their research boundaries to include human subject investigations in natural environments. For example, club drug use is associated with increased risk of HIV transmission; this association is particularly strong in men who have sex with men (MSM) [1]. Unfortunately, the concept of "club drug use" is neither simple nor well understood since illicit drug formulations often contain pharmacologically active agents known as "adulterants". These substances, whose presence is unknown to users, may produce undesirable pharmacologic and behavioral effects $[2,3]$. To modulate these effects, MSM often take "coingestants" [4-9]. The use of multiple substances by MSM in high-risk environments is common; even individuals who intend to use a single drug such as "ecstasy" may often receive greater than one substance because of the presence of adulterants. 
We appreciate that an exploration of the relationship between the substance use and HIV transmission risk might not be viewed by most as "toxicology research". Nonetheless, our investigation combines several areas of toxicologic expertise common such as adverse effects of illicit drugs and pharmaceuticals, drug interactions, and laboratory detection of substances. These skills are distinct from those of many NIH-funded drug abuse investigators whose backgrounds are in sociology, psychology, and other behavioral sciences. Their success - and, more importantly, their representation in NIH scientific review committeessuggests strongly that medical toxicologists should adopt their research methods. The purpose of this manuscript is to provide medical toxicologists with a proven research methodology that can be applied to substance abuse investigations. A secondary aim is to provide successful grant language that can be used in subsequent applications for research funding.

\section{Background of the Research}

Adulterants, drugs, and coingestants affect human physiology and, consequently, sexual behavior and HIV risk. For example, "ecstasy"- especially when adulterated with methamphetamine - can produce impotence, preventing MSM from performing insertive anal intercourse [2]. In highly sexualized environments, these men may transition to receptive anal sex, a behavior that dramatically increases HIV transmission risk [2]. To prevent these pharmacologic effects, some MSM paradoxically use Viagra as an HIV prevention measure because it promotes erection, facilitates use of a condom, and allows penetrative anal sex [2, 3, 10, 11]. Viagra also facilitates increased numbers of sexual contacts, longer periods of sexual activity, and intergenerational sex, features that may increase the risk of HIV transmission [3, 12-16].

Little is known about the phenomenon of drug, adulterant, and coingestant use. Because adulterants' presence is unknown, surveys cannot examine the selfreport of their use. Similarly, the coingestants used by MSM, or reasons for their use, are poorly understood. Because of the effect of substance use on social behaviors and HIV risk, an understanding of drug-related behaviors requires that we know - not infer- exactly what drugs were used. Since an exact knowledge of used substances requires gold standard analytical techniques, such as gas chromatography/mass spectroscopy (GC/MS), laboratory methods are critical to understanding the nature and effects of drug use by MSM in high-risk environments.

This study was important because it would develop a foundation for understanding the nature of drug, adulterant, and coingestant use, as well as their impact on social behavior. This line of investigation addressed a problem of public health importance, the prevention of HIV transmission. It was innovative because it compared HIV risk behaviors against biological standards of drug use, and the application of analytical laboratory science was a departure from existing social science methodology and represented a creative approach to behavioral science research.

\section{Sampling Method}

The University of Massachusetts Medical School, Tufts Medical School, and Fenway Community Health Institutional Review Boards approved all methods outlined below for advertising; obtaining verbal informed consent; enumerating venues; screening, recruiting, and interviewing study participants; and collecting specimens. Because we obtained information about illegal behaviors, the study was protected by a Certificate of Confidentiality.

We sampled MSM attending high-risk venues in Provincetown, MA, from the beginning of the Memorial Day weekend to the end of the Labor Day weekend over three successive years using a method known as the Young Mens' Survey [17]. Initially, the research team intended to (a) obtain prevalence of men who met inclusion criteria (Stage I); (b) select sampling frames-times and locations that could yield a sufficient number of MSM who might participate in the research (Stage II); and (c) from these sampling frames, select random sampling events where we could sample random MSM to provide a urine specimen and complete a interviewer-delivered survey (Stage III). We analyzed, in Stage IV, all urine specimens for a battery of 1,043 different drugs, adulterants, and coingestants using GC/MS. We intended to obtain 300 urine specimens and completed surveys per year (600 total for the study).

The goal of these relatively complex venue-based methods was to obtain a representative sample of MSM attending venues in Provincetown in whom we could examine drug use and risky HIV transmission behaviors. The sampling strategy for this study comprises the following three stages:

1. Identifying venues (Stage I): venues frequented by MSM in Provincetown, MA, were to be identified in the months prior to field work. These locations included dance clubs, bars, parties, parks (including beaches and docks), business establishments, and social organizations. Strategies to identify these venues and the days and times they are frequented include examining local publications, reviewing print and online publications and travel catalogs marketed to MSM, interviewing community informants and key public health officials, examining Provincetown, MA, 
Chamber of Commerce data, conducting focus groups, and the like. To assist in venue identification and subsequent recruitment, we hired a field research coordinator who summered in Provincetown. After the universe of possible venues in Provincetown, MA, was identified, an initial subset of venues was to be selected for further evaluation in Stage II.

2. Establishing sampling frames (Stage II): sampling frames are monthly lists of venues and their associated sampling periods. We intended to use multiple sampling periods, on different days of the week, for each venue to better describe the clientele and, hence, eligibility of a venue during a sampling frame [18]. (For example, a bar might have the following three sampling periods: Thursday evenings from 6-10PM and Friday and Saturday evenings from 9PM-1AM.). Only venues with at least one sampling period were to be included in the sampling frames. Because of the occurrence of seasonal parties and events in Provincetown, the identification of venues and the establishment of sampling frames require an ongoing, dynamic process.

To determine the eligibility of venues and the sampling periods, research teams comprising two to three research assistants (RAs) were to attend a venue. One member was to systematically count all men who appeared to be over the age of 18 at a defined intercept point during a 30-60-min period known as an enumeration period. Counting the number of men entering the venue during the sampling period is important because the primary clientele in some Provincetown, MA, bars transitions from MSM to lesbians during evening hours.

The second team member was to approach and administer a screening survey to men entering the venue. We used the following eligibility criteria for the screening survey that was administered during Stage II:

- Male

- able to speak or read English

- $\quad$ stated age of 18 or older

- able to consent to participation in the study

- has not previously participated in the study

The purpose of the RA-administered intercept screening survey was to collect demographics (age, race), a 5-day history of recreational substance use, venue-attendance information, and whether the respondent had had sex with a man in the last 12 months. Moreover, to prevent multiple enrollments, the screening survey identified whether the respondent had participated in the study previously. The screening survey consisted of seven items, contained no skip patterns, and required approximately $1-2 \mathrm{~min}$ for completion.
To estimate attendance, we had intended to conduct at least two 30-60-min enumeration periods at different times within each 4-h period. By multiplying the average number of persons counted by the percentage of persons counted who are eligible, standardized to a 4-h period, we would obtain an attendance estimate. At the end of the Stage II, a list of venues with estimates of MSM attendance and estimates of eligible men attending would be constructed.

3. Venue-based, cross-sectional sampling (Stage III): the sampling of venues entails a three-step process. In the first and second step, the venue and associated sampling periods, a set, are selected. The set is then used to schedule sampling events on a calendar for the upcoming weeks. In the third step, respondents were to be enrolled from venues in accordance with the sampling event calendar. We used the following inclusion criteria:

- Male

- able to speak or read English

- $\quad$ stated age of 18 or older

- able to consent to participation in the study

- has not previously participated in the study

Individuals to provide a urine specimen and complete the survey in Stage III met the additional inclusion criterion:

- must have had sex with a man within the last 12 months - must have used a club drug in the previous 5 days

In accordance with the sampling calendar, study staff counted, recruited, and obtained urine specimens and administered the survey to MSM. To conduct each sampling event, the field research team rented office space on a main street in Provincetown that could accommodate up to three concurrent individuals who are completing surveys. The use of an office also provided a confidential location to provide urine specimens, as well as a place to secure the study materials. During the entire sampling event in the first year of fieldwork, one team member systematically counted all men entering the defined intercept point; the remaining team members consecutively administered the screening survey to men at the intercept point. Men who stated that they have had sex with a man in the last 12 months and had used club drugs in the previous 5 days were eligible for further participation in the study by providing a urine specimen and completing the survey interview. Individuals who had previously participated in Phase III were ineligible.

We avoided multiple enrollments in the following way. We identified, by means of a check-off box, respondents who had participated in the study previously [17]. We had anticipated that the remuneration offered in the study 
offered insufficient incentive for potential respondents to enroll multiple times; as an additional protection, we rotated members of the research team so that visual identification of potential repeaters could be performed. Men in Provincetown tended to travel in groups, a social feature that enhanced our ability to spot potential repeaters.

We therefore collected the following data: (a) the total number of men; (b) the number of men who completed the survey; (c) age, race, whether the respondent had sex with a man in the last 12 months, sexual identity, and club drug use in the last 5 days; (d) the number who were determined to be eligible for the first time; and (e) the number of MSM who enrolled in the study. These data were used to determine enrollment rates.

Other recruitment procedures The RAs logged every participant using non-identifying descriptors (e.g., respondent 001,002 , etc) and recorded a final status on each individual (enrolled, refused to participate, did not meet eligibility criteria, could not urinate, etc.). The RAs estimated race/ethnicity of those who declined to participate in the study in order to track characteristics of those who refused. To minimize refusal, we offered an incentive $(\$ 20$ plus participation in a raffle for an iPhone). To every person contacted by our study staff, we offered a "goodie bag" containing a study flyer, addresses of HIV prevention services, condom, male lubricant, and an amusing candy such as Atomic Fireballs. Condoms and other prevention supplies such as lubricant were available at all recruiting sites as well as at the office. Finally, to enhance recruitment, our RAs included several MSM of varying ages and body types.

Urine specimen collection and storage After obtaining verbal informed consent, team members gave to respondents a urine specimen cup numerically linked to the survey interview and the screening form. Any respondent who was unable or unwilling to provide a urine specimen was excluded from the study. We provided respondents with specimen containers, gloves, fluid-impermeable containers such as plastic zipper bags (e.g., clinical laboratory "biohazard bags") and instructions on providing a midstream, clean-catch urine specimen. Because some illicit substances are rectally administered and the population under study may engage in unprotected, insertive anal sex, we avoided cross-contamination from sexual partners' drugs by requesting that all respondents use a cleansing wipe prior to urination.

We double-bagged all urine specimens and stored them at $-20^{\circ} \mathrm{C}$ until analysis could be completed. Frozen specimens were transported by study staff from Provincetown, MA, to Worcester, MA, on a weekly basis for analysis.
Analysis of specimens Frozen specimens were transported to the University of Massachusetts Medical School Drug Concentration Laboratory for analysis using gas chromatography/ mass spectroscopy using a Hewlett Packard Gas Chromatograph Mass Selective Detector model 5973/75 using a $0.2 \mathrm{~mm}$, $12 \mathrm{~m}, 0.33 \mu \mathrm{m}$ cross-linked methylsilicone GC column.

For analyses, $5 \mathrm{cc}$ of each participant's urine was pipetted into a glass extraction tube and the internal standard chlomipramine added, neutralized with ammonium hydroxide, and extracted with methylene chloride. After separation, the aqueous layer was extracted and the organic phase evaporated under nitrogen. The residue was then solubilized in $0.5-15 \mu 1$ of ethyl acetate.

We are capable of detecting 1,043 different drugs of abuse, pharmaceuticals, and dietary supplements by urine-sample test results against a computerized library, TOX1. We defined a match as agreement between spectra of $70 \%$ or greater. We also allowed a lower baseline for detection to ensure detection of minute amounts of analyte. We did not establish any deadlines for this completion of analyses, an allowance that permitted laboratory staff to complete analyses during slow work periods. We were, therefore, able to negotiate a price of only $\$ 15$ per run, a substantial cost saving over regular laboratory fees.

Modifications to the study protocol We refined our recruiting protocol from information gleaned during pilot testing in the summer of 2006. For example, we learned that many Provincetown vacationers stay from Saturday to Saturday; while most recruiting occurred in the later part of the week (e.g., Friday), most drug use occurred on the first Saturday night. Constraining the inclusion criteria to drug use in the last 5 days therefore eliminated a substantial number of potential participants. By increasing the time-from-lastdrug-use to 7 days, we substantially improved recruiting without sacrificing our ability to detect substances in urine. In addition, we questioned the validity of men who would admit, during screening intercepts, to using only marijuana while denying club drug use. During interviews, however, a substantial proportion of these men revealed broader drug use. We therefore modified the range of drugs used in the previous week to include marijuana. We also ascertained that nearly every location in Provincetown, MA, met our original criteria for a venue from which men could be recruited. We therefore treated the entire municipality of Provincetown as a venue, a modification that allowed men to be screened, recruited, and interviewed from a single location on the main thoroughfare of Provincetown, Commercial Street. Furthermore, we learned that a cohort of men who visited Provincetown simply stayed in guesthouses while using drugs and engaging in sex with partners recruited from Manhunt.com. We therefore developed an 
IRB-approved, Provincetown-specific study advertisement that was displayed on Manhunt during recruiting times; this banner advertisement listed the current study location and invited men to participate. Finally, we learned that the most effective recruiting periods were during morning hours. Evening recruiting hours interfered with nighttime social events, while men tended to visit beaches during afternoons. By focusing on morning recruiting periods, we were able to realize substantial increases in accrual.

\section{Results}

We approached 19,795 men between Memorial Day and Labor Day during 2007 and 2008. Of this number, 11,571 refused to be screened (59\%). Of the 8,224 men who agreed to screening, $6,499(81 \%)$ were ineligible. The $1,725(18 \%)$ who met inclusion criteria included 665 who agreed to participate in the interview and to provide a urine specimen. The demographic profile of study participants is presented in Table 1 . Approximately $17 \%$ of our study population described themselves as being HIV-positive. Approximately $53 \%$ of respondents engaged in oral sex after using drugs while visiting Provincetown, while only $13 \%$ of men reported engaging in more risky behaviors such as unprotected penetrative or receptive anal sex. Interestingly, we identified no difference in drug use or HIV risk behaviors between themed weeks (e.g., circuit party weekends) and non-themed weeks.

We detected 79 discreet substances in the urine of participants (Table 2). Almost 55\% of participants reported using sildenfil, vardenafil, or tadalafil, even though only $23 \%$ reported ever having erectile dysfunction. Notably, $20 \%$ of participants ingested sildenfil, vardenafil, or tadalafil specifically to modify the effect of club drugs. Furthermore, $35 \%$ of participants reported ingesting a substance with the specific intent of altering adverse effects from club drugs. Interestingly, we identified the presence of levamisole in the urine of an individual who had abused cocaine.

\section{Discussion}

We present a proven, feasible method for obtaining information on drug use patterns and associated behaviors in natural environments by hidden populations, individuals who engage in illegal or stigmatized behaviors and who are difficult to reach by standard sampling methods. Examples of hidden populations include users of illegal drugs and men who have sex with men [19]. Standard probability sampling methods, if applied to hidden populations, are
Table 1 Demographic characteristics $(n=665)$ : Project Apollo Survey Participants, 2007 and 2008

\begin{tabular}{|c|c|c|}
\hline & $\begin{array}{l}2007(n=311) \\
\text { Sample, \% }\end{array}$ & $\begin{array}{l}2008(n=354) \\
\text { Sample, \% }\end{array}$ \\
\hline Age, years & Median $=40$ & Median $=38.50$ \\
\hline $18-24$ & 9.7 & 10.7 \\
\hline $25-29$ & 13.2 & 18.1 \\
\hline $30-34$ & 11.9 & 11.9 \\
\hline $35-39$ & 13.2 & 12.3 \\
\hline $40-44$ & 18.8 & 14.4 \\
\hline $45-49$ & 19.0 & 14.8 \\
\hline $50-54$ & 9.7 & 11.1 \\
\hline $55-59$ & 2.6 & 4.5 \\
\hline $60-64$ & 1.3 & 1.7 \\
\hline $65-69$ & 0.3 & 0.3 \\
\hline $70-74$ & 0.3 & 0.3 \\
\hline \multicolumn{3}{|l|}{ Ethnicity } \\
\hline Hispanic & 8.7 & 9.3 \\
\hline Non-Hispanic & 91.3 & 90.7 \\
\hline \multicolumn{3}{|l|}{ Race } \\
\hline Asian & 0.97 & 1.5 \\
\hline Black/African American & 3.6 & 4.7 \\
\hline White & 86.4 & 87.1 \\
\hline American Indian/Alaskan Native & 0.65 & 0.88 \\
\hline Native Hawaiian/Pacific Islander & 0.32 & 0.59 \\
\hline Multi-racial & 2.9 & 5.3 \\
\hline Other & 5.2 & NA \\
\hline Residency status & & 30.5 \\
\hline Provincetown & 26 & 69.5 \\
\hline Tourist & 74 & 39.5 \\
\hline Massachusetts & 36.3 & 60.5 \\
\hline Non-Massachusetts & 63.7 & 30.5 \\
\hline \multicolumn{3}{|l|}{ Education } \\
\hline High school/some HS & 11.3 & 9.3 \\
\hline College/some college & 57.4 & 58.5 \\
\hline Graduate school & 31.3 & 32.2 \\
\hline
\end{tabular}

prohibitively costly because their subjects lack a sampling frame, have privacy concerns, and constitute a small part of the general population [19]. Traditional survey sampling methods such as telephone- or mail-administered surveys require a stable delivery endpoint; a survey delivered by either method would fail because vacationers lack a stable geographical address and often are not directly reachable by landline telephones. Similarly, recruitment only in venues where drug use is expected to occur (e.g., dance clubs) would likely introduce age bias into the sample population. Venue-based sampling, therefore, offers sufficient precision to obtain a representative sample if biases in study design can be identified and corrected. The importance for medical 
Table 2 Seventy-nine discreet substances identified in urine samples: Project Apollo Survey Participants, 2007 and 2008

\begin{tabular}{llll}
\hline Acetaminophen & Diazepam & Lidocaine & Pentoxifylline \\
Alprazolam & Diclofenac & MDA & Phenobarbitol \\
Amphetamine & Dihydrocdeine & MDMA & Phenylpropanolamine \\
Atomoxetine & Diltiazem & Meprobamate & Phenyltoloxamine \\
Atropine & Diphenhydramine & Methamphetamine & Phenytoin \\
Bupropion & Doxylamine & Methylphenidate & Propoxyphene \\
Butalbital & Ephedrine/pseudoephedrine & Metoclopramide & Quinine/quinidine \\
Caffeine & Flecainide & Metoprolol & Sertraline \\
Cannabinoids & Fluconazole & Mirtazapine & Sildenafil \\
Carbamazepine & Fluoxetine & Morphine & Theophylline \\
Chlorpheniramine & Gemfibrozil & Naproxen & Ticlopidine \\
Citalopram & Hydrocodone & Nevirapine & Topirmate \\
Cocaine & Hydromorphone & Nicotine & Tramadol \\
Cocaethyline & Hydroxyzine & Nordiazepam & Trazodone \\
Cotinine & Ibuprofen & Norpropoxyphene & Trimethoprim \\
Codeine & Imipramine & Nortriptyline & Venlafaxine \\
Cyclobenzaprine & Ketamine & Olanzapine & Zidovudine \\
Desipramine & Lamotrigine & Oxcodone & Zolpidem \\
Desmethyldiazepam & Lavamisole & Oxymorphone & Zopiclone \\
Dextromethorphan & Levetiracetam & Paroxetine & \\
\hline & & &
\end{tabular}

toxicologists is that venue-based sampling represents a method for obtaining representative samples of drug users who are distinct from those individuals seen in acute care environments, poison control centers, and other practice locations of medical toxicologists.

As a social science-derived research protocol, venuebased sampling was originally designed to obtain information via questionnaires requiring approximately $5 \mathrm{~min}$ for completion [18]. The leading innovation of our study protocol-and the aspect that makes this methodology relevant to medical toxicologists - is the rigorous analysis of biological materials to validate self-reported survey results. As physicians, medical toxicologists are capable of analyzing biological materials, as well as identifying acceptable methods for obtaining, storing, transporting, and analyzing biological specimens. Furthermore, medical toxicologists are qualified to identify the ways in which an analyte can produce drug-drug, drug-host, and drugenvironment interactions.

One advance contained in this investigation is the validation of self-reported club drug use in which a single person describing their activity (e.g., they "self-report" drug use), often without objective verification. Investigations that determine levels and patterns of illegal drug use frequently rely heavily upon self-report data collection methods, even though self-reported data is susceptible to distortion as respondents minimize behaviors that are undesirable, or highlight those that are acceptable [20]. To reduce this threat to our data, we selected a study population with high educational levels and sufficient means to travel, both features that support accurate self- reporting of drug use behaviors [20]. In addition, respondents were avidly engaged in the research question. Surveys were anonymous and were administered in a secure, confidential environment, and the incentive for study participation was too small to lead respondents to misrepresent their drug use or behaviors. We therefore had confidence that our study design offered few opportunities for respondents to misrepresent self-reported drug use.

Determining validity, however, involves comparing selfreported behaviors against some measure that is presumably more accurate [20]. Validation of self-reported drug use has involved comparison against standard urine drug of abuse screens ("toxic screens") [20]. Unfortunately, the toxic screen suffers from considerable false positive and false negative results, particularly among drugs used in club environments [21]. In our study, the use of GC/MS to detect a large number of substances was important for several reasons. First, respondents cannot self-report the use of adulterants, because the presence of adulterants is by definition unknown; we therefore needed a generalizable laboratory analytical method to detect these substances. Second, GC/MS required no confirmatory testing [21]. Finally, GC/MS, in the eyes of behavioral scientists, represents a significant advance simply because of the sheer numbers of substances that it can detect.

One important aspect of this study was to identify the presence of adulterants, those substances unknowingly ingested by study respondents. Adulterants represent an additional risk to the health of drug users, either from drug interactions or from direct toxicity. In our study sample, we identified the unknowing use of levamisole. Used as an 
antihelminthic and in combination cancer therapy, levamisole has been identified recently as an adulterant in recreational drugs [22]. The use of levamisole-adulterated substances has been associated with severe agranulocytosis and opportunistic infections [22]. We believe that our identification of levamisole supports this approach of using a rigorous laboratory method to identify the presence of adulterants, particularly those that are not targets of normal laboratory detection protocols.

We recognize that our specific research question represents a departure from many toxicological investigations and may have limited applicability to medical toxicologists. We nonetheless believe that exposure to this research methodology is important. First, many toxicologists are already familiar with the effects of acute drug overdose, making substance abuse research an academic arena that is easily accessible. Second, both the Society for Academic Emergency Medicine and the American College of Emergency Physicians have dedicated efforts to expanding the ranks of NIH-funded clinician-scientists in the field of emergency medicine - efforts that mirror those of individual departments of emergency medicine that seek to recruit and develop young clinician-scientists with the preparation and capability of achieving NIH funding. Third, as clinicians with board certification in an ACGME-approved subspecialty, medical toxicologists should be consistently performing at the highest academic levels. By adapting rigorous research methodologies, such as the variant of a venue-sampling technique described here, medical toxicologists can increase the breadth of research investigations amenable to funding and, therefore, heighten our academic profile. Finally, similar research methods (e.g., snowball sampling) have been used to extend the reach of emergency department interventions beyond the confines of the medical center. Medical toxicologists who have direct patient contact may consider developing similar research thrusts to identify greater numbers of individuals with uncommon substance abuse practices.

Acknowledgment This research was supported by NIH grant R01 DA-18572 (Boyer, PI) and in part by NIH grant 5K24AT003683 (Hibberd, PI)

\section{References}

1. Mattison A, Ross M, Wolfson TDF (2001) Circuit party attendance, club drug use, and unsafe sex in gay men. J Subst Abuse 13:119-126
2. Zemishlany Z, Aizenberg D, Weizman A (2001) Subjective effects of MDMA ('Ecstasy') on human sexual function. Eur Psychiatr 16(2):127-130

3. Halkitis P, Parsons J, Stirratt M (2001) A double epidemic: crystal methamphetamine drug use in relation to HIV transmission among gay men. J Homosex 41:17-35

4. Tong T, Boyer EW (2002) Club drugs, smart drugs, raves, and circuit parties: an overview of the club scene. Pediatr Emerg Care $18: 216-218$

5. Colfax G, Mansergh G, Guzman R, Vittinghoff E (2001) Drug use and sexual risk behavior among gay and bisexual men who attend circuit parties: a venue-based comparison. J Acquir Immune Defic Syndr 28:373-379

6. Boyer EW (2003) Poisoning data. From Massachusetts Poison Control Center

7. Kraner JC, McCoy DJ, Evans MA, Evans LE, Sweeney BJ (2001) Fatalities caused by the MDMA-related drug paramethoxyamphetamine (PMA). J Anal Toxicol 25(7):645-648

8. Shannon M (2000) Methylenedioxymethamphetamine. Pediatr Emerg Care 16(5):377-380

9. Case P (2003) Personal communication regarding results from RO1 DA-12334: HIV risk and club drugs among MSM: a two city comparison.

10. Anonymous (1996) New drugs for HIV infection. Med Lett 38:35-37

11. Frosch D, Shoptaw S, Huber A, Rawson R, Ling W (1996) Sexual HIV risk among gay and bisexual male methamphetamine abusers. J Subst Abuse Treat 13:483-486

12. Paul J, Stall R, Crosby G, Barrett D, Midanik L (1994) Correlates fo sexual risk taking among gay male substance users. Addiction 89:971-983

13. Kim A, Kent C, Klausner J (2002) Increased risk of HIV and sexually transmitted disease transmission among gay/bisexual men who use Viagra, San Francisco 2000-2001. AIDS 16:1425-1427

14. Sherr L, Bolding G, Maguire M, Elford J (2000) Viagra use and sexual risk behaviour among gay men in London. AIDS 14:20512052

15. Chu P, McFarland W, Gibson S et al (2003) Viagra use in a community-recruited sample of men who have sex with men, San Francisco. J Acquir Immune Defic Syndr 33:191-193

16. MacKellar D, Valleroy L, Karon J, Lemp G, Janssen R (1996) The Young Men's Survey: method for estimating HIV seroprevalence and risk factors among young men who have sex with men. Public Health Rep 111:138-144

17. Valleroy L, MacKellar D, Karon J, Rosen D, McFarland W, Shehan D (2000) HIV prevalence and assoicated risks in young men hwo have sex with men. JAMA 284:198-204

18. Muhib F, Lin L, Stueve A et al (2001) A venue-based methods for sampling hard-to-reach populations. Public Health Reports 116 (1):216-222

19. Harrison L, Hughes A (1997) The Validity of self-reported drug use: improving the accuracy of survey estimates. In: Harrison $\mathrm{H}$ (ed) NIDA Research Monograph 167. NIDA, Washington, DC, pp 1-16

20. Shaw L (2001) The clinical toxicological laboratory: contemporary practice of poisoning evaluation. AACC, Washington, DC

21. Kinzie E (2009) Levamisole found in patients using cocaine. Ann Emerg Med 53:546-547

22. Zhu N, LeGatt D, Turner R (2009) Agranulocytosis after consumption of cocaine adulterated with levamisole. Ann Intern Med 150:287-289 